\title{
Inverted sessile serrated polyp diagnosed by magnifying image-enhanced colonoscopy
}

Recently, improved endoscopic imaging and advancements in diagnostic technology, such as magnifying colonoscopy and image-enhanced endoscopy (IEE), including narrow-band imaging (NBI) systems, have provided a higher rate of detection of superficial and small colorectal tumors. Even a depressed colon cancer as small as $5 \mathrm{~mm}$ in size can be correctly diagnosed as submucosal deeply invasive carcinoma with magnifying chromoendoscopy and then appropriately treated surgically without endoscopic resection [1]. Furthermore, magnifying chromoendoscopy can differentiate between colorectal neoplastic and non-neoplastic polyps [2]. We report a case of a depressed lesion, about $8 \mathrm{~mm}$ in diameter, which was diagnosed as an inverted sessile serrated polyp (SSP) by magnifying image-enhanced colonoscopy before removal.

A 63-year-old man underwent surveillance colonoscopy at our department. Colonoscopy revealed a flat elevated polyp with a central depression, about $8 \mathrm{~mm}$ in diameter, in the ascending colon (Paris classification IIa and IIc) ( $\bullet$ Fig. 1).

No meshed capillary vessel (type I capillary pattern according to Sano's classification) was detected on the surface of the polyp by NBI with magnification (॰ Fig. 2) [3].

After the dye-spraying of $0.4 \%$ indigo carmine, a depressed area was clearly defined in the polyp. However, the pit pattern of the depressed area was not clearly observed because of the overlying dense mucus ( $\bullet$ Fig. 3).

Magnification with chromoendoscopy using $0.05 \%$ crystal violet staining after water washing showed a "dilated" typeII pit pattern in the depressed area (๑ Fig. 4) [4].

Based on the above endoscopic findings, an inverted SSP was suspected. Endoscopic resection was performed for histological evaluation. The polyp was completely removed en bloc with endoscopic mucosal resection (the lift and cut technique) without complication. A histological diagnosis of inverted SSP was finally established ( $\bullet$ Fig. 5).

Inverted growth of a hyperplastic polyp is characterized by epithelial misplacement or inversion of the epithelium into the

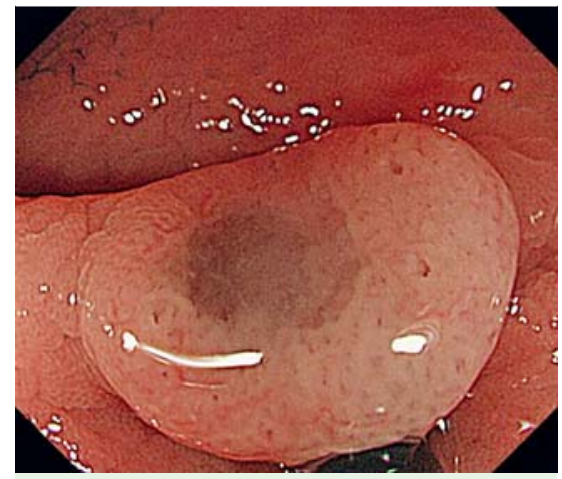

Fig. 1 Colonoscopy revealed a flat elevated polyp with a central depression, about $8 \mathrm{~mm}$ in diameter, in the ascending colon.

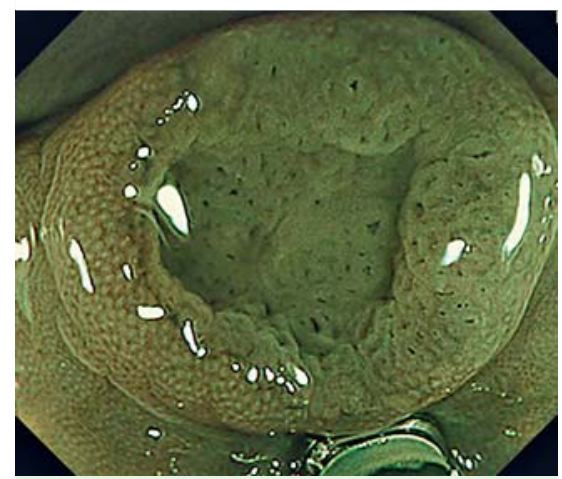

Fig. 2 Narrow-band imaging with magnification did not show meshed capillary vessels (type-I capillary pattern according to Sano's classification).

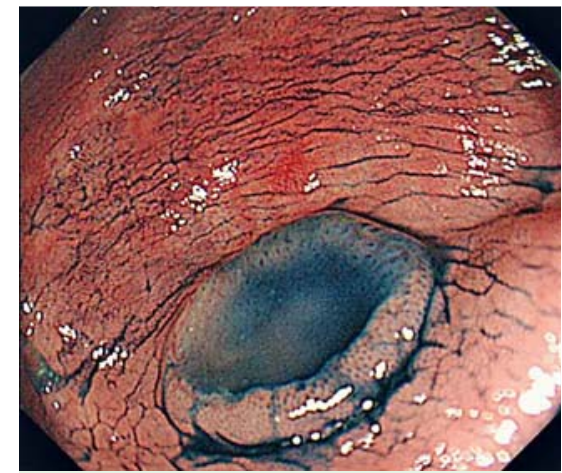

Fig. 3 After the dye-spraying of $0.4 \%$ indigo carmine, a depressed area was clearly defined in the polyp. However, the pit pattern of the depressed area was not clearly observed because of the overlying dense mucus.

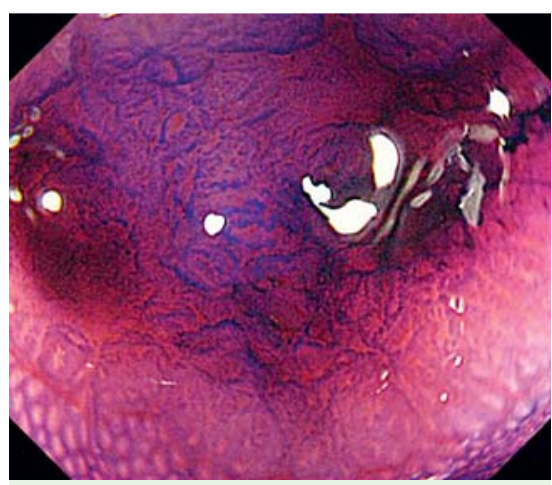

Fig. 4 Magnification with chromoendoscopy using $0.05 \%$ crystal violet staining after water washing showed a "dilated" type-Il pit pattern in the depressed area.

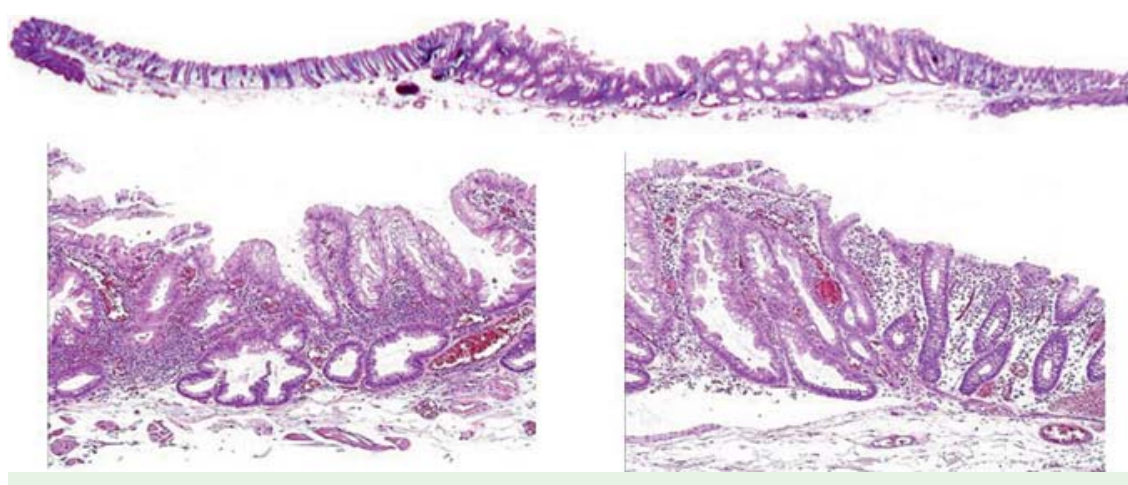

Fig. 5 Histological examination revealed serrated tubular features in the depressed portion, some of which showed the elements of extension and divergence. The muscularis mucosa was rising at the both ends of the lesion and it was unclear in the depressed portion. A histological diagnosis of inverted sessile serrated polyp was finally established.

submucosa, which is a variant form of an exophytic hyperplastic polyp [5]. Owing to the inverted growth pattern, a depres- sed area is present in such lesions, which could be misdiagnosed as an early colorectal neoplasm on conventional view. Our 
case, however, was correctly diagnosed as an inverted SSP before removal, because we applied magnifying IEE for histological prediction. A “dilated" type-II pit pattern could be an endoscopic hallmark of an SSP, which is different from a hyperplastic polyp with normal type-II pit pattern.

Endoscopy_UCTN_Code_CCL_1AD_2AB

\section{Competing interests: None}

\section{T. Muramoto ${ }^{1}$, Y. Oono ${ }^{1}$, K.-I. Fu²,} H. Ikematsu ${ }^{1}$, T. Yano ${ }^{1}$, T. Kojima ${ }^{1}$, K. Minashi' ${ }^{1}$, K. Kaneko ${ }^{1}$

1 Division of Digestive Endoscopy and Gastrointestinal Oncology, National Cancer Center Hospital East, Chiba, Japan

2 Department of Gastroenterology, Juntendou University Nerima Hospital, Tokyo, Japan

\section{References}

1 Nakajima T, Saito Y, Matsuda T et al. Minute depressed-type submucosal invasive cancer-5 $\mathrm{mm}$ in diameter with intermediate lymph-node metastasis: report of a case. Dis Colon Rectum 2007; 50: 677-681

2 Fu KI, Sano Y, Kato S et al. Chromoendoscopy using indigo-carmine dye-spraying with magnifying observation. Is the most reliable method for differential diagnosis between non-neoplastic and neoplastic colorectal lesions? A prospective study. Endoscopy 2004; 36: 1089-1093

3 Sano Y, Ikematsu H, Fu KI et al. Meshed capillary vessels using narrow band imaging for differential diagnosis of small colorectal polyps. Gastrointest Endosc 2008; 69: 278 283

4 Kudo S, Hirota S, Nakajima T et al. Colorectal tumors and pit pattern. J Clin Pathol 1994; 47: $880-885$

5 Sobin $L$. Inverted hyperplastic polyps of the colon. Am J Surg Pathol 1985; 9: 265-272

\section{Bibliography}

Dol $10.1055 / \mathrm{s}-0030-1256377$

Endoscopy 2011; 43: E201 -E202

(c) Georg Thieme Verlag KG Stuttgart · New York . ISSN 0013-726X

\section{Corresponding author}

\section{T. Muramoto, MD}

Department of Gastroenterology National Cancer Center Hospital East

6-5-1 Kashiwanoha

Kashiwa City

Chiba 277-8577

Japan

Fax: +81-471-346928

takashi_mura7711@hotmail.com 